\title{
Analysis of Folded Erbium-Doped Planar Waveguide Ampliers by the Method of Lines
}

\author{
Weibin Huang and Richard R. A. Syms
}

\begin{abstract}
A numerical model for spiral and folded erbiumdoped planar waveguide amplifiers has been developed, based on a rate equation model of the local complex dielectric constant and beam propagation by the method of lines. A five-level system is used to describe the ion-ion interactions that occur at high erbium concentrations. A suitable form of the method of lines is presented in polar coordinates, and absorbing boundary conditions based on the third-order rational series approximation are derived. Using this model, amplification in both straight and curved slab guides can be simulated, and examples of propagation in typical folded amplifier structures are presented.
\end{abstract}

Index Terms - Erbium-doped planar waveguide amplifier (EDWA), method of lines, modeling, optical amplifier, rate equation.

\section{INTRODUCTION}

I $\mathrm{N}$ recent years, erbium-doped planar waveguide amplifiers (EDWA's) have received attention for gain at $1535 \mathrm{~nm}$, because of the potential for integration with pump lasers and filters. Suitable techniques for forming the glass layers include sputtering [1], [2], FHD and PECVD [3]-[6], and the sol-gel process [7]-[10]. Useful gains have already been reported; for example, Schmulovich et al. reported a reduction of loss on pumping of $8.75 \mathrm{~dB} / \mathrm{cm}$ in a straight erbium-doped sodium calcium silicate guide in 1992 [1].

Theoretical models of erbium-doped silica were devised for fiber amplifiers [11]. More recent models include effects that degrade performance as the erbium concentration rises [12]-[17]. These have been adapted to describe the extremely high doping levels of planar EDWA's; however, attention has focussed more on rate equation modeling than propagation. Most authors use either the finite element [14]-[15] or the variables-separation method [17], and solve the wave equation for a straight guide.

Layouts containing curved guides with small bend radii will be needed to minimize the size of planar EDWA's. For example, Fig. 1(a) shows a spiral geometry, which includes bends of different radii and right-angle intersections to achieve a long path in a compact chip. Here, differences in shading indicate consecutive sections of different curvature. Fig. 1(b) is similar to Fig. 1(a), but the intersections are eliminated through the use of a central fold-back bend.

Manuscript received August 10, 1998; revised July 20, 1999. This work was supported in part by the European Commission via ACTS Project AC047: CAPITAL.

The authors are with the Department of Electrical and Electronic Engineering, Optical and Semiconductor Devices Section, Imperial College of Science, Technology and Medicine, London SW7 2BT U.K.

Publisher Item Identifier S 0733-8724(99)09690-5.
Curved waveguides cannot guide electromagnetic fields without radiation loss. Furthermore, the transverse fields of both the pump and the signal beam are distorted in bend sections, so that the spatial overlap between the two fields and the erbium-doped region is lowered. Both these effects make the normal models inaccurate for folded amplifiers. Bending loss in amplifiers has been examined using a phenomenological treatment based on a local absorption coefficient [18]. However, a rigorous theory such as the method of lines (MoL) [19] is potentially more appropriate. Not only can it describe both the effects above, but it can also model losses arising from changes in curvature or waveguide proximity effects in a unified manner.

Suitable MoL algorithms have been developed for passive guides with straight [19], uniformly curved [20] and arbitrarily curved [21] geometries. In the last of these, the structure was modeled by a piecewise linear approximation. Here, a variant of the MoL is introduced for active guides in polar coordinates. Folded amplifiers are then modeled by cascading sections of appropriate curvature.

The complex refractive index of a uniform gain medium is first introduced in Section II, based on prior models. The imaginary component describes absorption and gain for the pump and signal waves, and is obtained by rate-equation modeling. A five-level model is used to account for upconversion effects at high erbium doping levels, and the relevant rate equations are solved numerically.

In Section III, the MoL is reviewed, and it is shown how the wave equation may be solved in polar coordinates. Calculation of the local complex dielectric constant is then linked to a beam propagation algorithm for the pump and signal waves [11]. In Section IV, absorbing boundary conditions [22]-[24] based on the third-order rational series approximation are developed for polar coordinates. Solutions for typical geometries involving curved and folded slab guides are then presented in Section V, and it is shown that excessive curvature and curvature changes both reduce the overall gain more than would be expected simply from consideration of radiation loss.

\section{ABSORPTION AND GAIN IN UNIFORM MEDiA}

We first review the conventional rate equation model for gain in a uniform glass host containing a dispersion of erbium ions [11]. EDWA's are based on a three-level system, with ${ }^{4} \mathrm{I}_{15 / 2},{ }^{4} \mathrm{I}_{13 / 2}$ and ${ }^{4} \mathrm{I}_{11 / 2}$ being the ground, metastable and pump level (levels 1, 2 and 3, respectively). Absorption of pump radiation at $980 \mathrm{~nm}$ wavelength promotes electrons from level 


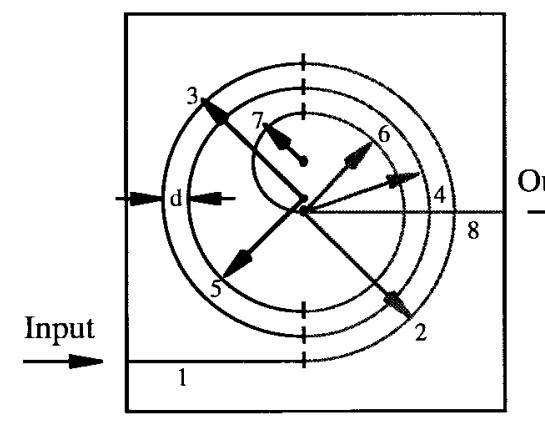

(a)

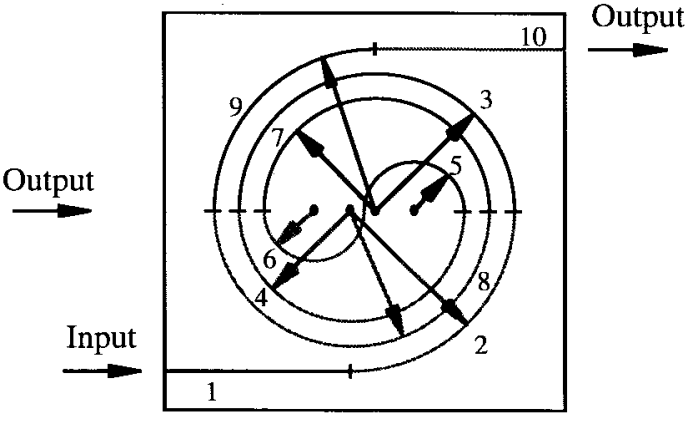

(b)

Fig. 1. Geometries for planar EDWA's: (a) spiral and (b) folded spiral.

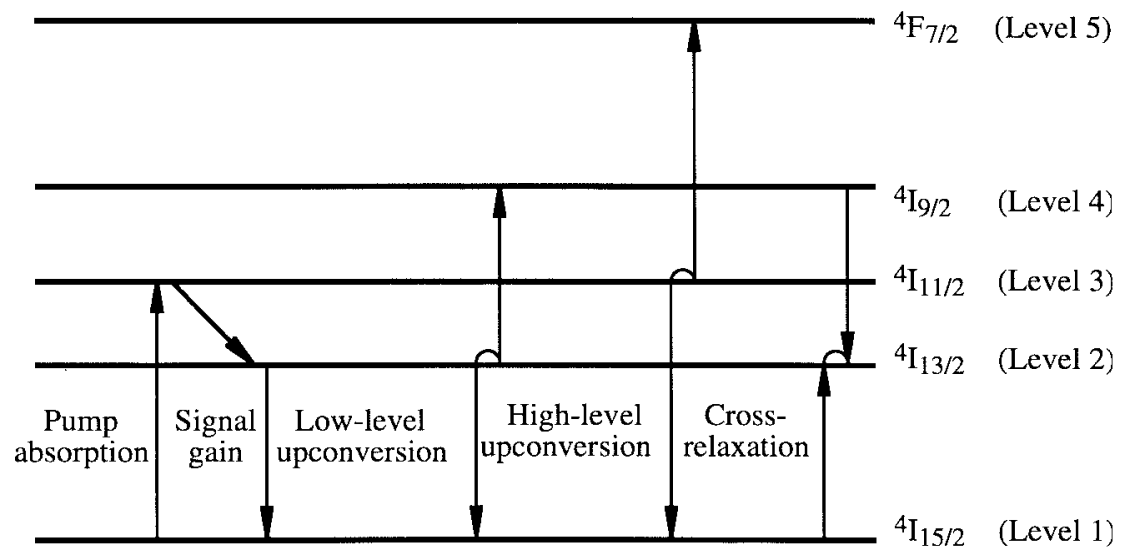

Fig. 2. Simplified energy level diagram for $\mathrm{Er}^{3+}$ (after [15]).

1 to level 3. After decaying to level 2, these electrons provide signal gain at $1535 \mathrm{~nm}$ via transitions to level 1 (Fig. 2).

A very high $\mathrm{Er}^{3+}$ concentration is needed in EDWA's. Unfortunately, at densities above $10^{25} \mathrm{~m}^{-3}$, ion-ion interactions allow nonradiative energy transfer between near-neighbors, reducing the population of the metastable level [15]-[17]. Here, up-conversion and cross-relaxation effects involving ${ }^{4} I_{9 / 2}$ (level 4) and ${ }^{4} F_{7 / 2}$ (level 5) are considered. Suitable rate equations have been developed by several authors [12]-[17]. Here, we assume the following set:

$$
\begin{aligned}
d N_{1} / d t= & -\sigma_{13} N_{1} v_{g} N_{p}+\left\{\sigma_{21} N_{2}-\sigma_{12} N_{1}\right\} v_{g} N_{s} \\
& +N_{3} / \tau_{31}+N_{2} / \tau_{2}+K_{2} N_{2}^{2}+K_{3} N_{3}^{2}-C_{14} N_{1} N_{4} \\
d N_{2} / d t= & \left\{\sigma_{12} N_{1}-\sigma_{21} N_{2}\right\} v_{g} N_{s} \\
& -N_{2} / \tau_{2}+N_{3} / \tau_{32}-2 K_{2} N_{2}^{2}+2 C_{14} N_{1} N_{4} \\
d N_{3} / d t= & \sigma_{13} N_{1} v_{g} N_{p} \\
& -N_{3} / \tau_{31}-N_{3} / \tau_{32}-2 K_{3} N_{3}^{2}+N_{4} / \tau_{4} \\
d N_{4} / d t= & K_{2} N_{2}^{2}-C_{14} N_{1} N_{4}-N_{4} / \tau_{4}+N_{5} / \tau_{5} \\
d N_{5} / d t= & K_{3} N_{3}^{2}-N_{5} / \tau_{5}
\end{aligned}
$$

Here $N_{1}, N_{2} \cdots N_{5}$ are the densities of levels $1,2 \cdots 5$, respectively, and sum to $N_{e r}$, the $E r^{3+}$ ion density. Similarly, $N_{p}$ and $N_{s}$ are the photon densities for the pump and signal beams. The constants are as follows. The cross-section for pump absorption is $\sigma_{13}$; the corresponding coefficient $\sigma_{31}$ for pump emission is assumed to be zero. The spontaneous decay path from level 3 is described by a lifetime $\tau_{32}$. A further path
TABLE I

COEFFICIENTS OF ERBIUM IONS USED IN THE Numerical Simulations (After [12], [15], [16])

$$
\begin{aligned}
& \lambda_{\mathrm{s}}=1535 \mathrm{~nm} \\
& \lambda_{\mathrm{p}}=980 \mathrm{~nm} \\
& \sigma_{12}=5.36 \times 10^{-25} \mathrm{~m}^{2} \\
& \sigma_{21}=5.41 \times 10^{-25} \mathrm{~m}^{2} \\
& \sigma_{13}=2.58 \times 10^{-25} \mathrm{~m}^{2} \\
& \sigma_{31}=0 \times 10^{-25} \mathrm{~m}^{2}
\end{aligned}
$$$$
\tau_{2}=1 \times 10^{-2} \mathrm{sec}
$$$$
\tau_{31}=2 \times 10^{-1} \mathrm{sec}
$$$$
\tau_{32}=1 \times 10^{-5} \mathrm{sec}
$$$$
\tau_{4}=1 \times 10^{-9} \mathrm{sec}
$$$$
\mathrm{K}_{2}=\mathrm{K}_{3}=3.5 \times 10^{-24} \mathrm{~m}^{3} / \mathrm{sec}
$$$$
\mathrm{C}_{14}=3.5 \times 10^{-24} \mathrm{~m}^{3} / \mathrm{sec}
$$

from level 3 to level 1 has a lifetime $\tau_{31}$. The cross sections for signal absorption and emission are $\sigma_{12}$ and $\sigma_{21}$, respectively. $\tau_{2}, \tau_{4}$ and $\tau_{5}$ are lifetimes for levels 2,4 and 5, respectively; $K_{2}$ and $K_{3}$ are upconversion coefficients, and $C_{14}$ is a crossrelaxation coefficient. Suitable numerical values are given in Table I [12], [15], [16]. $v_{g} \approx c / \sqrt{ } \varepsilon_{r}^{\prime}$ is the group velocity, where $c$ is the velocity of light and $\varepsilon_{r}^{\prime}$ is the relative dielectric constant of the host glass, assumed real so that background losses are ignored. Note that $N_{p}$ and $N_{s}$ are the only photon densities in (1). Modification of the rate equations to include amplified spontaneous emission through the addition of extra photon terms is simple, but is not considered necessary for the present demonstration. 
The quantities $N_{p}$ and $N_{s}$ are related to the time-averaged electric fields $E_{p}$ and $E_{s}$ of the pump and signal beams. The irradiances $I_{p}$ and $I_{s}$ of the two beams are

$$
I_{p, s}=N_{p, s} v_{g} h c / \lambda_{p, s}
$$

where $h$ is Planck's constant, and $\lambda_{p}$ and $\lambda_{s}$ are the wavelengths of the pump and signal. However, in an electromagnetic model of plane waves, the irradiances would be given by

$$
I_{p, s}=1 / 2 \sqrt{ }\left\{\varepsilon_{0} \varepsilon_{r}^{\prime} / \mu_{0}\right\}\left|E_{p, s}\right|^{2}
$$

$N_{p}$ and $N_{s}$ may therefore be found by equating (2) and (3). Equations (1) may then be solved in the steady state by combining the lower two equations to eliminate $N_{5} / \tau_{5}$. For given values of $N_{p}$ and $N_{s}$, the densities $N_{1} \ldots N_{4}$ may then be found by solving the remaining equations using, e.g., Newton's method, assuming that $N_{5}$ is negligible because of the short lifetime of level 5 .

The rate equations may be linked with a propagation model by assuming that pump and signal beams decay and grow (say) in the $s$-direction according to $d I_{p} / d s=-a_{p} I_{p}$ for the former and $d I_{s} / d_{s}=g_{s} I_{s}$ for the latter, where $a_{p}$ and $g_{s}$ are power absorption and gain coefficients given by

$$
a_{p}=\sigma_{13} N_{1} \text { and } g_{s}=\sigma_{21} N_{2}-\sigma_{12} N_{1} .
$$

Alternatively, the medium may be described by a complex permittivity, in which the coefficients of (4) contribute an imaginary susceptibility to the host glass. The complex relative dielectric constants $\varepsilon_{r p}$ and $\varepsilon_{r s}$ of the doped material at the pump and signal wavelengths are then [11]

$$
\varepsilon_{r p}=\varepsilon_{r}^{\prime}-j\left(\sqrt{ } \varepsilon_{r}^{\prime}\right) a_{p} / k_{0 p}
$$

and

$$
\varepsilon_{r s}=\varepsilon_{r}^{\prime}+j\left(\sqrt{ } \varepsilon_{r}^{\prime}\right) g_{s} / k_{0 s}
$$

where $k_{0 p}=2 \pi / \lambda_{p}$ and $k_{0 s}=2 \pi / \lambda_{s}$. Equation (5) may then be used in the model of guided wave propagation developed in the following sections.

\section{The Method of Lines FOR ACtive WAVEGUIDES IN POLAR COORDINATES}

We now develop a model of propagation in folded EDWA's. We assume that the circuit is constructed by cascading $n$ sections of uniformly curved waveguide bend, as shown in Fig. 1 . The $i$ th section has a radius $R_{i}$ and an arc length $s_{i}$. The waveguide is assumed to be a slab of erbium-doped glass of width w, with complex relative dielectric constants $\varepsilon_{r p}$ and $\varepsilon_{r s}$ for the pump and signal waves. Outside the core, the glass is undoped, with a real relative dielectric constant $\varepsilon_{r}^{\prime \prime}$.

Each section is modeled in isolation, by considering propagation in a finite window of width $\mathrm{W}$ (less than the guide separation d in Fig. 1). Fig. 3(a) and (b) show windows for straight and curved sections. Both may be modeled using the MoL in polar coordinates, with appropriate radii. We, therefore, now give a brief review of the MoL [19].

Propagation of a transverse electric (TE)-polarized field in polar coordinates is described by the Helmholtz wave equation, $\partial^{2} E / \partial r^{2}+1 / r \partial E / \partial r+1 / r^{2} \partial^{2} E / \partial \phi^{2}+k_{0}^{2} \varepsilon_{r} E=0$, where $E$ is the electric field, $\varepsilon_{r}$ is a spatially varying relative dielectric constant, and $k_{0}=2 \pi / \lambda$. Introducing new variables $x=r-R$ and $s=R \phi$, where $R$ is the radius of curvature, this reduces to

$$
\partial^{2} E / \partial s^{2}+\gamma^{2} \partial^{2} E / \partial x^{2}+C \gamma \partial E \partial x+\gamma^{2} k_{0}^{2} \varepsilon_{r} E=0
$$

where $\gamma=1+C x$, and $C=1 / R$. When $C=0$, the Cartesian form of (6) is recovered.

The region is now discretised in the $x$-direction into a set of $m$ lines spaced apart by a distance $\Delta x=W /(m-1)$. If this is done, we obtain the matrix-vector differential equation:

$$
d^{2} \underline{E} / d s^{2}+\left\{\underline{\gamma}^{2} \underline{P}+C \underline{\gamma} \underline{D}+\underline{\gamma}^{2} k \underline{\varepsilon}_{r}\right\} \underline{E}=0
$$

where $\underline{E}$ is a vector of size $m$ containing values of $E$ at each line, $\underline{\varepsilon}_{r}$ is an $m \times m$ diagonal matrix of similar values of $\varepsilon_{r}$, $\gamma$ is a diagonal matrix with elements $\gamma_{j j}=\left(1+C x_{j}\right)$, where $x_{j}$ is the coordinate of the $j$ th line, $\underline{D}$ is an $m \times m$ matrix representing the discretized first derivative with respect to $x$, namely,

$$
\underline{D}=1 / \Delta x\left[\begin{array}{ccccccc}
-1 & 1 & 0 & 0 & 0 & \cdots & 0 \\
0 & -1 & 1 & 0 & 0 & \cdots & 0 \\
0 & 0 & -1 & 1 & 0 & \cdots & 0 \\
\cdots & \cdots & \cdots & \cdots & \cdots & \cdots & \cdots \\
\cdots & \cdots & \cdots & 0 & 0 & 0 & -1
\end{array}\right]
$$

and $\underline{P}$ is an $m \times m$ tridiagonal matrix, representing the discretized form of the second derivative

$$
\underline{P}=1 / \Delta x^{2}\left[\begin{array}{ccccccc}
-2 & 1 & 0 & 0 & 0 & \cdots & 0 \\
1 & -2 & 1 & 0 & 0 & \cdots & 0 \\
0 & 1 & -2 & 1 & 0 & \cdots & 0 \\
\cdots & \cdots & \cdots & \cdots & \cdots & \cdots & \cdots \\
\cdots & \cdots & \cdots & 0 & 0 & 1 & -2
\end{array}\right] .
$$

If we now introduce a new matrix $Q$, such that $Q^{2}=$ $\gamma^{2} \underline{P}+C \gamma \underline{D}+\gamma^{2} k_{0}^{2} \underline{\varepsilon}_{r}$, (7) reduces to the second-order equation $d^{2} \underline{E} / d s^{2}+Q^{2} \underline{E}=0$. Solutions for constant $\underline{Q}^{2}$ then have the form

$$
\underline{E}(s)=\underline{T} \exp \{-j \underline{\beta} s\} \underline{T}^{-1} \underline{E}_{f}+\underline{T} \exp \{+j \underline{\beta} s\} \underline{T}^{-1} \underline{E}_{b} .
$$

Here $\beta$ and $\underline{T}$ are the eigenvalue and eigenvector matrices of $\underline{Q}$, respectively, so that $\underline{Q}=\underline{T} \underline{\beta} \underline{T}^{-1}$. Note that (10) contains both forward and backward waves, with amplitudes defined by $\underline{E}_{f}$ and $\underline{E}_{b}$. However, backward waves may normally be neglected in long, weakly guiding structures. Generally, $\underline{\varepsilon}_{r}$ is a function of $s$, so the matrix $Q^{2}$ must also vary. However, (10) may still give a solution over a short distance $\Delta s$ over which $\underline{\varepsilon}_{r}$ and $\underline{Q}^{2}$ are locally constant, in the form

$$
\underline{E}\left(s_{0}+\Delta s\right)=\underline{T} \exp \{-j \underline{\beta} \Delta s\} \underline{T}^{-1} \underline{E}\left(s_{0}\right) .
$$

Using (11), the solution may be propagated step-by-step through a slowly varying structure.

A model for curved, amplifying media may be developed by linking the algorithm above to the rate equation model as shown in Fig. 4. Pump and signal field vectors $\underline{E}_{p}$ and $E_{s}$ are first computed at the input by solving the eigenvalue equation for a passive slab guide of core width $\mathrm{w}$, and core and cladding indices $n_{\text {core }}=\sqrt{ } \varepsilon_{r}^{\prime}$ and $n_{\text {clad }}=\sqrt{ } \varepsilon_{r}^{\prime \prime}$, and then 


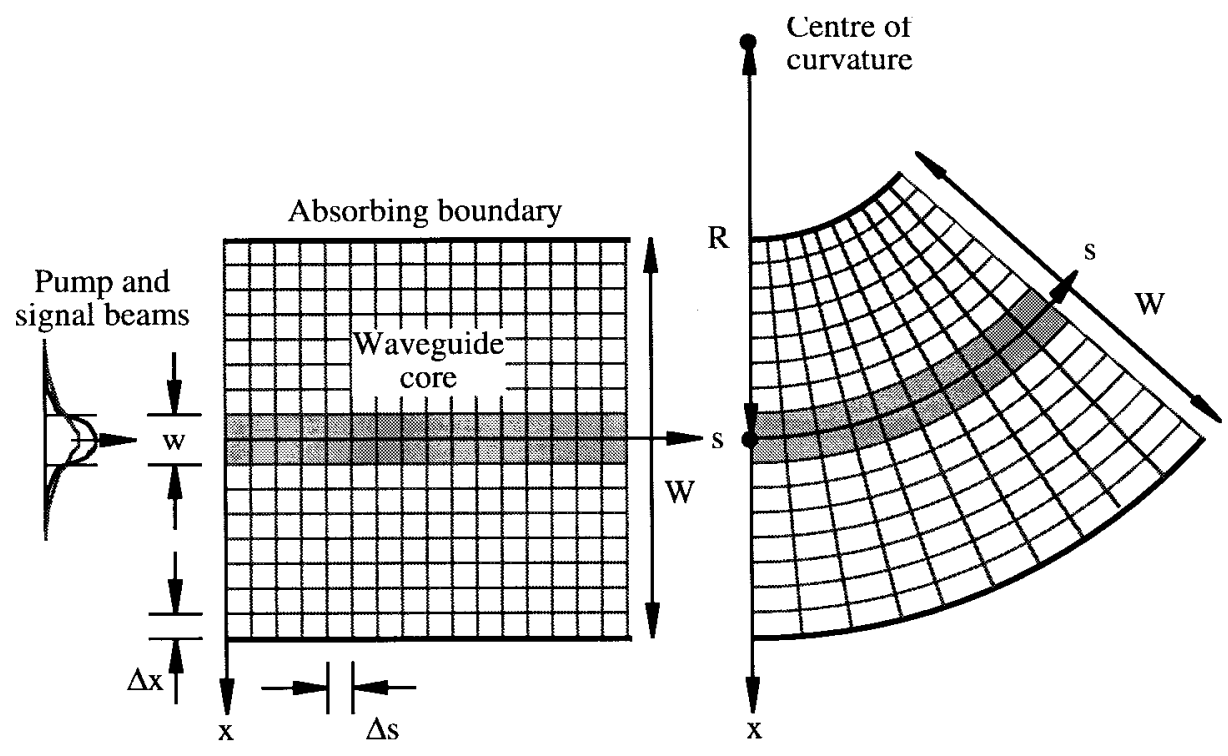

(a)

(b)

Fig. 3. Discretization used in the method of lines: (a) Cartesian coordinates and (b) polar coordinates.

discretising the resulting field functions. Two MoL algorithms, with separate matrices $\underline{Q}_{p}^{2}$ and $\underline{Q}_{s}^{2}$, are then used to propagate the pump and signal vectors through each section in turn. At each step within a bend, the photon densities $N_{p}$ and $N_{s}$ are first calculated from $\underline{E}_{p}$ and $\underline{E}_{s}$ for each line, and the corresponding values of $\underline{\varepsilon}_{r p}$ and $\underline{\varepsilon}_{r s}$ are used to construct matrices $\underline{Q}_{p}^{2}$ and $\underline{Q}_{s}^{2}$. These are decomposed into eigenvalue and eigenvector matrices $\underline{T}_{p, s}$ and $\underline{\beta}_{p, s}$ by standard methods, and (11) is used to propagate each vector by a distance $\Delta s$.

Further modification of the overall scheme could be carried out to incorporate amplified spontaneous emission (ASE). This would involve addition to the rate equations of a number of extra terms $N_{s e, i}$ (with $i=1 \ldots n$ ) describing the photon densities at a set of ASE wavelengths $\lambda_{s e, i}$ and use of separate MoL algorithms with corresponding matrices $\underline{Q}_{s e, i}^{2}$ to propagate each one.

\section{ABSORBING BOUNDARY CONDITIONS FOR POLAR COORDINATES}

Beam propagation algorithms suffer from the apparent reflection of diverging waves back into the calculation region, especially in folded circuits which naturally create radiation. Absorbing boundaries are therefore introduced to suppress these waves. Many authors have investigated boundaries based on factorization of the propagation operator. In [23], expressions for second- and third-order rational series approximations are derived. Dreher [24] and Pregla [22] adapted the second- and third-order versions to the MoL in Cartesian coordinates. Here, we develop the third-order terms for polar coordinates.

The approximation is derived as follows. With the notation $D_{s}^{2}=\partial^{2} / \partial s^{2}, D_{x}^{2}=\partial^{2} / \partial x^{2}, D_{x}=\partial / \partial x, L_{x}=\gamma D_{x}+C / 2$ and $\omega^{2}=\gamma^{2} k_{0}^{2} \varepsilon_{r}-C^{2} / 4$, (6) may be written as:

$$
L E=\left\{L_{x}^{2}+\omega^{2}+D_{s}^{2}\right\} E=0 .
$$

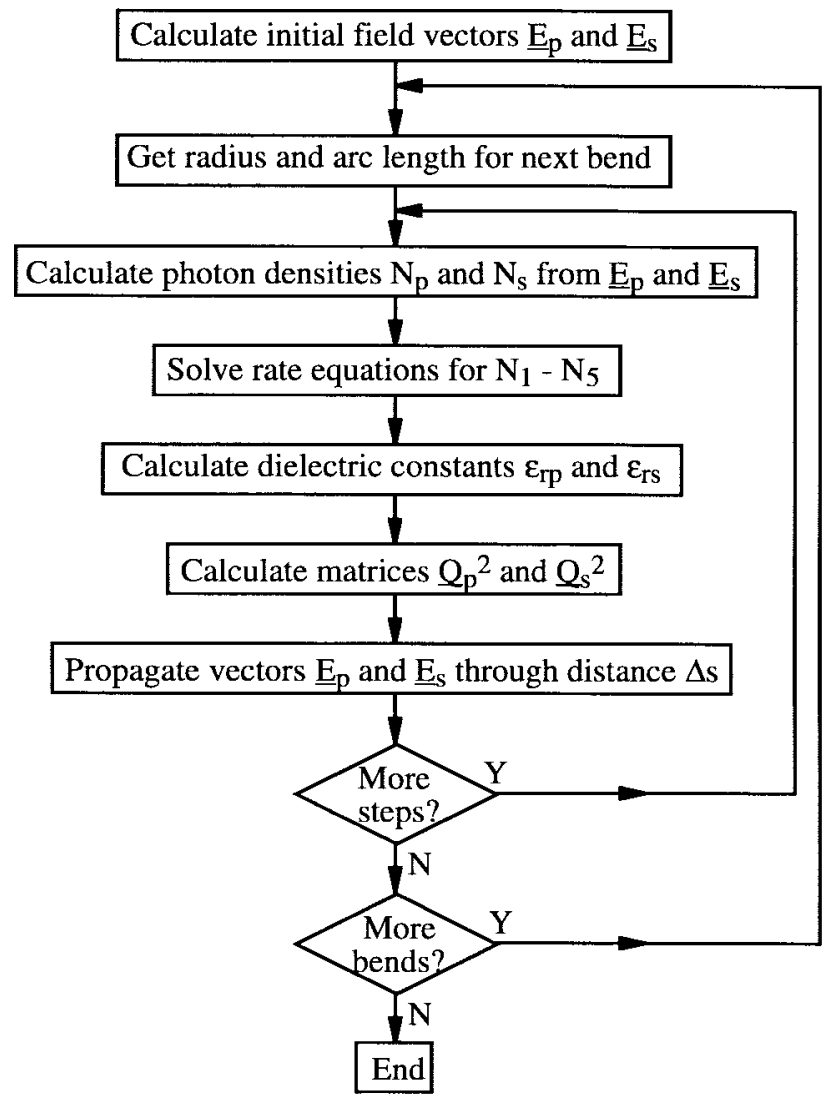

Fig. 4. Flowchart showing the linkage between the rate equation model and the method of lines.

Following [22], [23], we now factor the operator $L$ as $L=$ $L^{+} L^{-}$, where $L^{+}$and $L^{-}$are

$$
L^{ \pm}=L_{x} \pm j \omega \sqrt{ }\left\{1+D_{s}^{2} / \omega^{2}\right\}
$$

Here, the "+" and "-" signs correspond to waves in the $+x$ and $-x$ directions, respectively. At the upper boundaries in 
TABLE II

CoEfFicients of Rational SERIES ApProXimations for Absorbing Boundary Conditions (AFTER [23])

\begin{tabular}{llllll}
\hline Type & $\mathrm{p}_{0}$ & $\mathrm{p}_{2}$ & $\mathrm{q}_{0}$ & $\mathrm{q}_{2}$ & Angles of absorption $\left({ }^{\circ}\right)$ \\
\hline $\begin{array}{lllll}\text { Padé }^{\infty} \\
\mathrm{L}_{\mathrm{a}}\end{array}$ & 1.00000 & -0.75000 & 1.00000 & -0.25000 & 0.00 \\
Chebyshev & 0.99973 & -0.80864 & 1.00000 & -0.31657 & $11.7,31.9,43.5$ \\
Least squares & 0.99650 & -0.91296 & 1.00000 & -0.47258 & $15.0,45.0,75.0$ \\
Chebyshev-Padé & 0.99250 & -0.92233 & 1.00000 & -0.51084 & $18.4,51.3,76.6$ \\
Newman & 0.99030 & -0.94314 & 1.00000 & -0.55560 & $18.4,53.1,81.2$ \\
$\mathrm{~L}^{\infty}$ & 1.00000 & -1.00000 & 1.00000 & -0.66976 & $0.00,60.5,90.0$ \\
& 0.95651 & -0.94354 & 1.00000 & -0.70385 & $26.9,66.6,87.0$
\end{tabular}

Fig. 3, a suitable absorbing condition may be constructed by allowing only waves in the $-x$ direction, so that $L^{-} E=0$; similarly, we assume that $L^{+} E=0$ on the lower boundaries.

Using the third-order rational series approximation, we then write the square root in (13) as

$$
\sqrt{ }\left\{1+S^{2}\right\} \approx\left\{p_{0}+p_{2} S^{2}\right\} /\left\{q_{0}+q_{2} S^{2}\right\}
$$

where $p_{0}, p_{2}, q_{0}$, and $q_{2}$ are constants chosen to give exact agreement with the radix for particular values of $S$, and hence for particular angles of incidence on the boundaries. Values for common approximations are shown in Table II [23]. If this is done, the condition $L^{-} E=0$ becomes

$$
\left\{\left\{q_{0} \omega^{2}+q_{2} D_{s}^{2}\right\} L_{x}-j \omega\left\{p_{0} \omega^{2}+p_{2} D_{s}^{2}\right\}\right\} E=0
$$

Using (12), the term $D_{s}^{2}$ may be eliminated from (15), to give an equation of the form

$$
\left\{a_{3} \Delta x^{3} D_{x}^{3}+a_{2} \Delta x^{2} D_{x}^{2}+a_{1} \Delta x D_{x}+a_{0}\right\} E=0
$$

where the coefficients $a_{0} \ldots a_{3}$ are given by

$a_{0}=q_{2} C^{3} / 8-j \omega p_{2} C^{2} / 4+\omega^{2} C\left(q_{2}-q_{0}\right) / 2+j \omega^{3}\left(p_{0}-p_{2}\right)$

$a_{1}=3 q_{2} C^{2} g / 4-j \omega p_{2} C g+\omega^{2}\left(q_{2}-q_{0}\right) g$

$a_{2}=3 q_{2} C g^{2} / 2-j \omega p_{2} g^{2} \quad a_{3}=q_{2} g^{3}$

with $g=\gamma / \Delta x$. We now discretise (16) in the $x$-direction, and use the result to obtain modified elements in $Q^{2}$ that represent the absorbing boundary. To do so, we introduce a ficticious additional element $E_{0}$ just outside the window to the vector $\underline{E}$. In line 1 , we then use the discrete approximations $D_{x} E_{1} \approx$ $\left\{-1 / 6 E_{3}+E_{2}-1 / 2 E 1-1 / 3 E_{0}\right\} / \Delta x, D_{x}^{2} E_{1} \approx\left\{E_{2}-2 E_{1}+\right.$ $\left.E_{0}\right\} / \Delta x^{2}$ and $D_{x}^{3} E_{1} \approx\left\{E_{3}-3 E_{2}+3 E_{1}-E_{0}\right\} / \Delta x^{3}$, obtained from a Taylor series expansion, to reduce (16) to

$$
E_{0}=\alpha_{1} E_{1}+\alpha_{2} E_{2}+\alpha_{3} E_{3}
$$

where

$$
\begin{aligned}
& \alpha_{1}=\left\{3 a_{3}-2 a_{2}-a_{1} / 2+a_{0}\right\} /\left\{a_{3}-a_{2}+a_{1} / 3\right\} \\
& \alpha_{2}=\left\{-3 a_{3}+a_{2}+a_{1}\right\} /\left\{a_{3}-a_{2}+a_{1} / 3\right\} \\
& \alpha_{3}=\left\{a_{3}-a_{1} / 6\right\} /\left\{a_{3}-a_{2}+a_{1} / 3\right\}
\end{aligned}
$$

We may of course perform a similar manipulation of (12). We start by writing this as

$$
\left\{D_{s}^{2}+b_{2} \Delta x^{2} D_{x}^{2}+b_{1} \Delta x^{1} D_{x}+b_{0} \Delta x^{0}\right\} E=0
$$

where $b_{0}=\omega^{2}+C^{2} / 4, b_{1}=g C$ and $b_{2}=g^{2}$. We now perform a similar discretization on (20) using (18) and (19) to eliminate the unknown element $E_{0}$. We then obtain

$$
\begin{aligned}
D_{s}^{2} E_{1} & +\left\{g^{2}\left(\alpha_{1}-2\right)-g C\left(1 / 2+\alpha_{1} / 3\right)+\left(\omega^{2}+C^{2} / 4\right)\right\} E_{1} \\
& +\left\{g^{2}\left(1+\alpha_{2}\right)+g C\left(1-\alpha_{2} / 3\right)\right\} E_{2} \\
& +\left\{g^{2} \alpha_{3}-g C\left(1 / 6+\alpha_{3} / 3\right)\right\} E_{3}=0
\end{aligned}
$$

Equation (21) shows that the absorbing boundary conditions are represented by modifying the top row of the matrix $Q^{2}$ to contain the elements

$$
\begin{aligned}
& \underline{Q}^{2}(1,1)=g^{2}\left(\alpha_{1}-2\right)-g C\left(1 / 2+\alpha_{1} / 3\right)+\left(\omega^{2}+C^{2} / 4\right) \\
& \underline{Q}^{2}(1,2)=g^{2}\left(1+\alpha_{2}\right)+g C\left(1-\alpha_{2} / 3\right) \\
& \underline{Q}^{2}(1,3)=g^{2} \alpha_{3}-g C\left(1 / 6+\alpha_{3} / 3\right) .
\end{aligned}
$$

A similar procedure may be followed to find the elements on the bottom row of $Q^{2}$, this time assuming that $L^{+} E=0$. These results reduce to those of Pregla [22] for Cartesian coordinates when $C=0$. In the following section, we use conditions based on the $L_{a}^{\infty}$ approximation in Table II.

\section{NumericAl Results}

Simulations of slab guide amplifiers based on the algorithms of the preceding sections were performed using a window width $\mathrm{W}=40 \mu \mathrm{m}$, a line spacing $\Delta x=0.4 \mu \mathrm{m}$ and a step size $\Delta s=0.5-1.0 \mathrm{~mm}$. The guide was centrally placed within the window; the core width was $3.2 \mu \mathrm{m}$, and the core and cladding indices were 1.46 and 1.468 , respectively, so that the guide was just single-moded at $980 \mathrm{~nm}$. Transverse fields derived from the equivalent passive structure were launched at the input, and modal powers obtained after propagation were computed by overlap integrals with these fields.

Fig. 5 shows the variation of signal gain with distance for straight and uniformly curved EDWA's at an $\mathrm{Er}^{3+}$ concentration of $5 \times 10^{25} \mathrm{~m}^{-3}$, for input pump and signal intensities of 


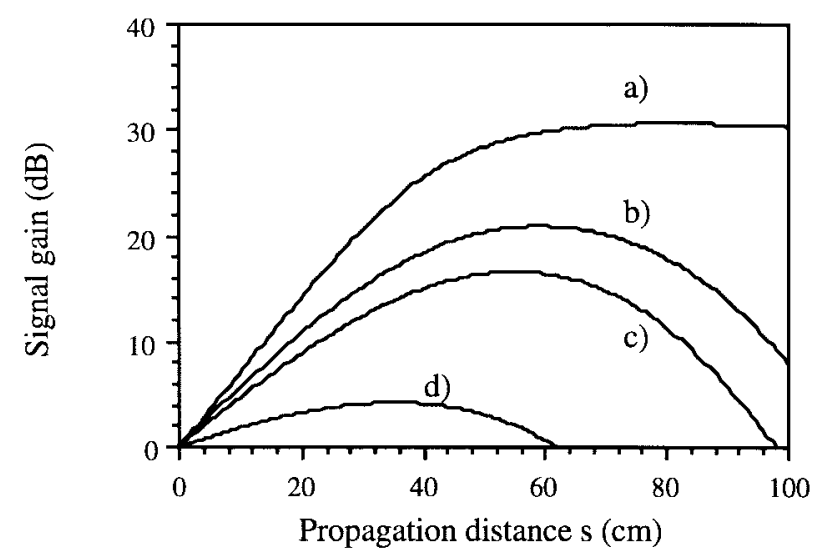

Fig. 5. Variation of signal gain with propagation distance for straight guides, (a) with and (b) without ion-ion interactions, and curved guides of radius (c) $5 \mathrm{~mm}$ and (d) $4.5 \mathrm{~mm}$.

$1 \mathrm{~mW}$ and $0.2 \mu \mathrm{W}$ per square micron, respectively, averaged over the core. Curve a) shows the gain obtained in a straight guide, in the absence of ion-ion interactions. Curve b) shows the corresponding result with ion-ion interactions included; the net effect is clearly to reduce the signal gain by depletion of the metastable level. Curves c) and d) show the gain variation in curved guides, for radii of $R=5 \mathrm{~mm}$ and $R=4.5$ $\mathrm{mm}$, respectively. In each case, and from now on, ion-ion interactions are also considered. The gain is clearly reduced very considerably as the radius is reduced to $4.5 \mathrm{~mm}$.

To investigate whether the gain reduction could be ascribed to radiation loss in the signal alone, numerical values for the gain near the amplifier input were compared with attenuation figures for similarly curved passive guide geometries. For curved amplifiers of radii $R=5 \mathrm{~mm}$ and $R=4.5 \mathrm{~mm}$, the gains were $0.0909 \mathrm{~dB} / \mathrm{cm}$ and $0.3673 \mathrm{~dB} / \mathrm{cm}$ lower than in a straight amplifier, respectively. However, the radiation loss in passive guides of the same radii were only $0.0745 \mathrm{~dB} / \mathrm{cm}$ and $0.3421 \mathrm{~dB} / \mathrm{cm}$. Thus, while the majority of the gain reduction is clearly due to radiation losses, an additional component must also be due to changes in the gain profile and its overlap with the signal.

For example, Fig. 6 shows the variation over the crosssection of the pump and signal field amplitudes (compared with a maximum of unity at the input) and the local gain coefficient $g_{s}$, after propagating a distance $3 \mathrm{~cm}$ in a curved guide of radius $5 \mathrm{~mm}$. Although the pump remains reasonably well confined, its peak has shifted slightly outward and the gain profile is clearly no longer symmetric. Due to its weaker confinement, the signal has radiated outward to a much greater degree.

The lateral range of this figure is shown smaller than the window width $\mathrm{W}$ for clarity. However, the efficacy of the absorbing boundary conditions was verified by the relative immunity of the field profiles to changes in either the value of $\mathrm{W}$ or the position of the core within that range.

The next two figures show simulations of the geometries of Fig. 1, and illustrate the ease with which the model may be used to investigate and optimize folded amplifier geometries. In each case, the interguide separation is $d=100 \mu \mathrm{m}$,

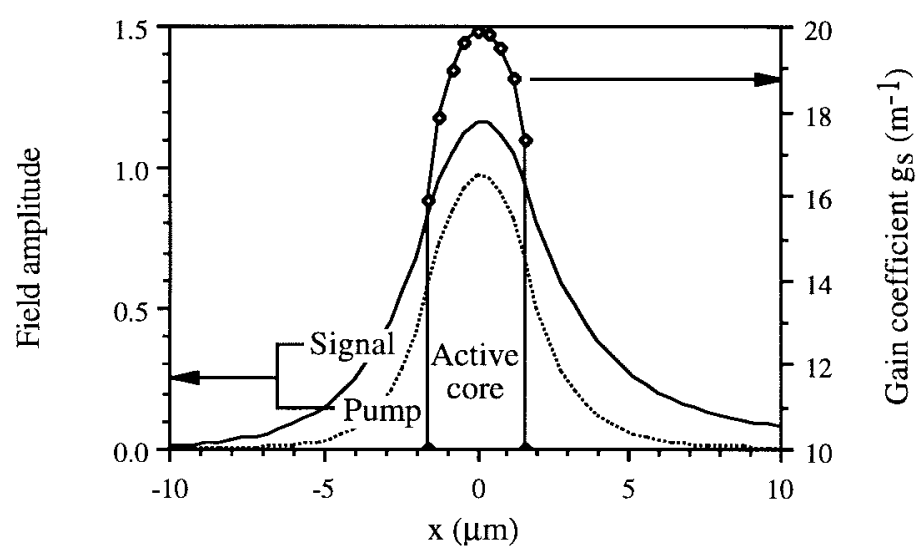

Fig. 6. Transverse variation of the pump and signal field amplitudes and the local gain coefficient $g_{s}$, for a curved guide with radius $5 \mathrm{~mm}$, after propagating a distance $3 \mathrm{~cm}$.

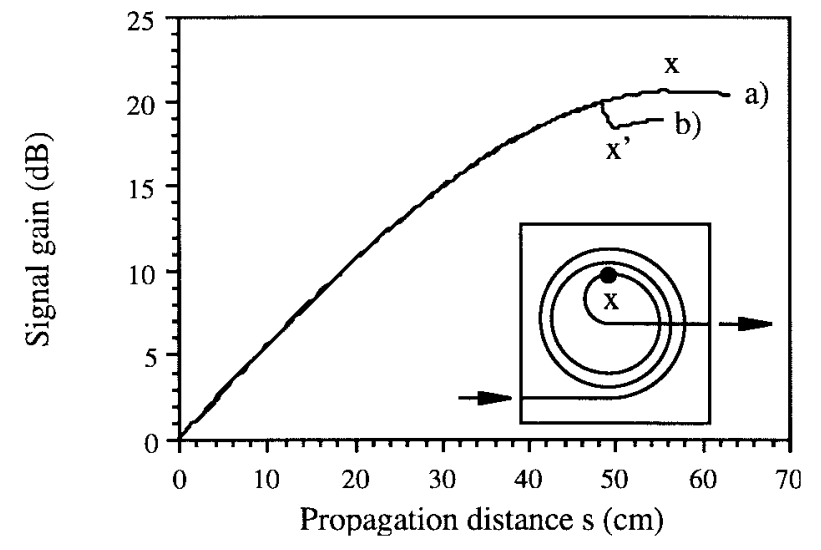

Fig. 7. Variation of signal gain with distance for the spiral amplifier of Fig. 1(a), with key radii of (a) $R_{6}=10 \mathrm{~mm}, R_{7}=5 \mathrm{~mm}$ and (b) $R_{6}=8.5$ $\mathrm{mm}, R_{7}=4.25 \mathrm{~mm}$.

and the input power densities are as before. Fig. 7 shows two simulations of the layout of Fig. 1(a); in Curve a), the two key radii are $R_{6}=10 \mathrm{~mm}$ and $R_{7}=5 \mathrm{~mm}$, while in Curve b) the corresponding values are $8.5 \mathrm{~mm}$ and $4.25 \mathrm{~mm}$. Values for other radii follow from the geometry, and the length of the straight guide sections at input and output was $2 R_{2}$. For Curve a), the gain rises smoothly to a maximum, and little reduction in gain occurs after the curvature discontinuity between Sections VI and VII (point $x$ ). For Curve b), considerable loss occurs after the corresponding junction (point $x^{\prime}$ ), illustrating the necessity of avoiding large curvatures if good performance is to be maintained.

This geometry includes waveguide intersections, which were simulated separately using the MoL in Cartesian coordinates. Due to the short propagation distance involved, the effect on the signal was found to be negligible, as described earlier by others for passive structures [25]. However, in practice stringent control on feedback is required to prevent oscillation occurring instead of amplification.

Fig. 8 shows simulations of the geometry of Fig. 1(b), which avoids intersections by the use of a central fold-back bend. Here, the key radii are $r_{6}=4.5 \mathrm{~mm}$ and $r_{7}=9.05 \mathrm{~mm}$. Fig. 8(a) shows results obtained with continuously aligned 


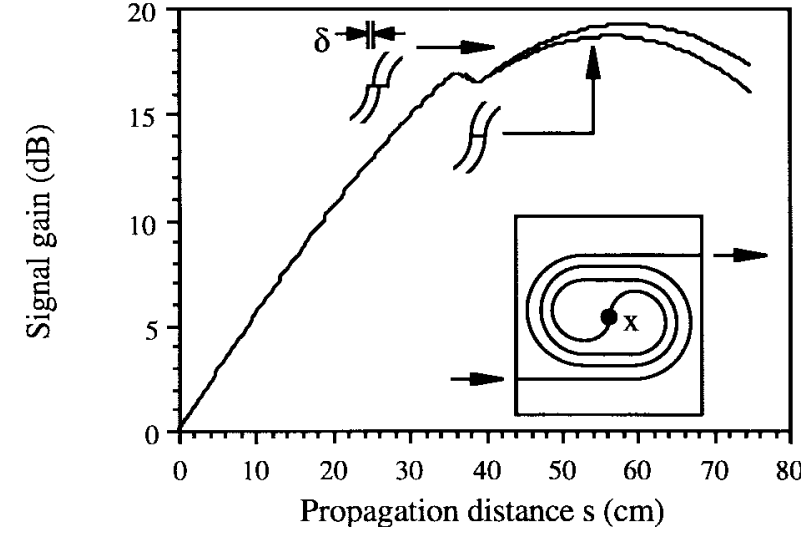

Fig. 8. Variation of signal gain with distance for the folded spiral amplifier of Fig. 1(b), with minimum radii of $R_{6}=4.5 \mathrm{~mm}, R_{7}=9.05 \mathrm{~mm}$ : (a) without and (b) with an offset central joint.

cores. There is again a significant reduction in gain at the junction between Sections VI and VII, this time due to the mismatch in mode position caused by curvature reversal. This excess loss can be reduced by the strategy developed for passive guides, namely the introduction of a lateral offset $\delta$ between the cores [26]. Fig. 8(b) shows the improvement obtained for $\delta=0.4 \mu \mathrm{m}$, which can be simulated very simply by altering the lateral position of the core within the window, to a resolution set by the discretization of the model.

\section{CONCLUSIONS}

A model for erbium-doped planar waveguide amplifiers has been presented, based on a combination of rate equation modeling and the method of lines. A five-level rate equation model is used to describe the conventional three-level system, together with up-conversion and cross-relaxation effects that occur at high erbium concentrations. The densities of the ground, pump and metastable states obtained obtained by solving these equations are then used to calculate a local complex dielectric constant for the pump and signal waves. The two waves are then propagated separately using a beam propagation algorithm based on the method of lines.

Using this model, amplification in both straight and curved guides can be simulated, and the effects of radiation loss and changes in overlap between the signal and the core gain distribution may be separated. We have shown how gain can be optimized in typical folded geometries using simple adjustments to the layout. The model could easily be extended to allow propagation of other waves, particularly an ASE signal.

\section{REFERENCES}

[1] J. Schmulovich, A. Wong, Y. H. Wong, P. C. Becker, A. J. Bruce, R. Adar " $\mathrm{Er}^{3+}$ glass waveguide amplifier at $1.55 \mu \mathrm{m}$ on silicon," Electron. Lett., vol. 28, pp. 1181-1182, 1992.

[2] R. N. Ghosh, J. Shmulovich, C. F. Kane, M. R. X. de Barros, G. Nykolak, A. J. Bruce, and P. C. Becker, "8-mW threshold $\mathrm{Er}^{3+}$-doped planar waveguide amplifier," IEEE Photon. Technol. Lett., vol. 8, pp. 518-520, 1996.

[3] T. Kitagawa, K. Hattori, K. Shuto, M. Yasu, M. Kobayashi, and M. Horiguchi, "Amplification in erbium-doped silica-based planar lightwave circuits," Electron. Lett., vol. 28, pp. 1818-1819, 1992.
[4] K. Shuto, K. Hattori, T. Kitagawa, Y. Ohmori, and M. Horiguchi, "Erbium-doped phosphosilicate glass waveguide amplifier fabricated by PECVD," Electron. Lett., vol. 29, pp. 139-141, 1993.

[5] K. Hattori, T. Kitagawa, M. Oguma, Y. Ohmori, and M. Horiguchi "Erbium-doped silica-based waveguide amplifier integrated with 980/1530 nm WDM coupler," Electron. Lett., vol. 30, pp. 856-857, 1994.

[6] K. Takeda, M. Oguma, H. Yamada S., Mitachi, and M. Golling "Gain distribution measurement of an erbium-doped silica-based planar waveguide amplifier using a complex OLCR," IEEE Photon. Technol. Lett., vol. 9, pp. 1102-1103, 1997.

[7] A. S. Holmes, R. R. A. Syms, K. Ueki, and H. Yanagawa, "Er-doped silica waveguide using sol-gel method," in Proc. 55th Autumn Meeting, Japan Soc. Appl. Phys., Nagoya, Japan, Sept. 19-22, 1994, paper 21P-R-5.

[8] X. Orignac and R. M. Almeida, "Silica-based sol-gel optical waveguides on silicon," Proc. Inst. Elect. Eng.-J. Optoelectron., vol. 143, no. 287, 1996.

[9] M. Benatsou and M. Bouazaoui, "Fluorescence properties of sol-gel derived $\mathrm{Er}^{3+}: \mathrm{SiO}_{2}-\mathrm{GeO}_{2}$ planar waveguides," Opt. Comm., vol. 137, pp. $143-150,1997$.

[10] A. Martucci, G. Brusatin, and M. Guglielmi, "Fabrication and characterization of sol-gel $\mathrm{GeO}_{2}-\mathrm{SiO}_{2}$ erbium-doped planar waveguides," Proc. 9th Int. Workshop on Glasses, Ceramics, Hybrids and Nanocomposites from Gels, Sheffield, U.K., 31 Aug.-5 Sept. 1997, Paper F5.

[11] E. Desurvire, Erbium Doped Fiber Amplifiers: Principles and Applications. New York: Wiley, 1994, pp. 215-225.

[12] P. Blixt, J. Nilsson, T. Carlnas, and B. Jaskorzynska, "Concentrationdependent upconversion in $\mathrm{Er}^{3+}{ }_{\text {-doped fiber amplifiers: Experiments }}$ and modeling," IEEE Photon. Technol. Lett., vol. 3, pp. 996-998, 1991.

[13] E. Delavaque, T. Georges, M. Monerie, P. Lamouler, and J.-F. Bayon, "Modeling of pair-induced quenching in erbium-doped silicate fibers," IEEE Photon. Technol. Lett., vol. 5, pp. 73-75, 1993.

[14] M. Federighi, I. Massarek, and P. F. Trwoga, "Optical amplication in thin optical waveguides with high Er concentration," IEEE Photon. Technol. Lett., 5, 227-229 (1993).

[15] F. Di Pasquale, M. Zoboli, M. Federighi, and I. Massarek, "Finite element modeling of silica waveguide amplifiers with high erbium concentration," IEEE J. Quantum Electron., 30, pp. 1277-1281, 1994.

[16] F. Di Pasquale and M. Federighi, "Modeling of uniform and pairinduced upconversion mechanisms in high-concentration erbium-doped silica waveguides," J. Lightwave Technol., vol. 13, pp. 1858-1864, 1995.

[17] O. Lumholt, T. Rasmussen, and A. Bjarklev, "Modeling of extremely high concentration erbium-doped silica waveguides," Electron. Lett., vol. 29 , pp. 495-496, 1993.

[18] M. Ohashi and K. Shiraki, "Bending loss effect on signal gain in an $\mathrm{Er}^{3+}$-doped fiber amplifier," IEEE Photon. Technol. Lett., vol. 4, pp. 192-194, 1992.

[19] R. Pregla and W. Pascher, "The method of lines" in Numerical Techniques for Microwave and Millimeter Wave Passive Structures, T. Itoh, Ed. New York: Wiley, 1989.

[20] R. Pregla, "The method of lines for the analysis of dielectric waveguide bends," J. Lightwave Technol., vol. 14, pp. 634-639, 1996.

[21] R. Pregla and E. Ahlers, "Method of lines for analysis of arbitrarily curved waveguide bends," Electron. Lett., vol. 30, pp. 1478-1479, 1994.

[22] R. Pregla, "MoL-BPM method of lines based beam propagation method," Progr. Electromagn. Res. PIER-11, pp. 51-102, 1995.

[23] T. G. Moore, J. G. Blaschak, A. Taflove, and G. A. Kriegsmann, "Theory and application of radiation boundary operators," IEEE Trans. Antennas Propagat., vol. 36, pp. 1797-1812, 1988.

[24] A. Dreher and R. Pregla, "Analysis of planar waveguides with the method of lines and absorbing boundary conditions," IEEE Microwave Guided Wave Lett., vol. 1, pp. 138-140, 1991.

[25] F. Mackenzie, C. J. Beaumont, M. Nield, and S. A. Cassidy, "Measurement of excess loss in planar silica X junctions," Electron. Lett., vol. 28, pp. 1919-1920, 1992.

[26] T. Kitoh, N. Takato, M. Yasu, and M. Kawachi, "Bending loss reduction in silica-based waveguides by using lateral offsets," J. Lightwave Technol., vol. 13, pp. 555-562, 1995.

Weibin Huang, photograph and biography not available at the time of publication.

Richard R. A. Syms, photograph and biography not available at the time of publication. 